\title{
THE PREVENTION OF INFECTIOUS DISEASES IN CHILDHOOD
}

By J. L. Henderson, M.D., F.R.C.P.E. James Mackenzie Professor of Child Health, University of St. Andrezus

The development of reliable methods of active immunization against the common infectious diseases in childhood is proving a difficult problem, but the recent isolation and propagation by tissue culture of the viruses of most of the common infectious diseases have brightened the prospect. Johnson and Goodpasture isolated the mumps virus in 1934. More recently Enders and his colleagues, also in the United States, have cultured the viruses of mumps (Habel, I945), poliomyelitis (Enders et al., I949), chicken pox (Weller, 1953) and measles (Enders et al., 1954), and Anderson (1954) in Australia has cultured the rubella virus. The preparation of vaccines for these diseases has thus become a possibility. Active immunization has proved effective and reliable in the prevention of smallpox, diphtheria and tetanus, and of considerable value in the attenuation, if not always the prevention, of whooping cough; but natural infection is still the only way of acquiring a lasting immunity to mumps, chicken pox, measles and rubella. No such immunity is conferred by such diseases as the common cold and influenza.

The purpose of this paper is to evaluate methods of active immunization employed against the common infectious diseases in this country.

\section{Smallpox}

The proportion of infants vaccinated in this country has declined considerably in this century, but there has been an increase in the last few years. In England and Wales the acceptance rate showed a fairly steady rise from 23.8 per cent. in 1950 to 34.2 per cent. in 1953 . It is highly desirable to encourage vaccination of the young infant by every available means, because more people travel abroad than ever before and rapid air communication with countries in which smallpox is endemic increases the risk of outbreaks in this country. Moreover, the possibility of postvaccinial complications, notably encephalitis, is less when primary vaccination is performed in infancy. The best age for vaccination is betweet one and six months, after the neonatal period has been successfully passed, but before the lesion is: likely to be damaged by the activity of the child

\section{Diphtheria}

The policy of routine immunization in earl childhood introduced by the Ministry of Healtg in 1940 has been most successful, for diphtheria has almost been eliminated in this country. The mortality in England and Wales, which average $2,86 \mathrm{I}$ per annum in the quinquennium 1935-39.0 had fallen to an average of 29 per annum in gूf quinquennium 1950-54. In 1954 only nine persons died from the disease. The proportion of immsine children has always varied with the efficiency. of the local authority services and has recently de clined in some areas. This trend, which probablo reflects the impression that diphtheria no longe? constitutes a menace, is alarming and must b $\overrightarrow{\vec{E}}$ vigorously resisted, for it is believed that the in 3 cidence of diphtheria will rise if the proportion of immune children falls much below the presen level of about 70 per cent. An interesting althoug more indirect result of immunizing a high pro.portion of children has been a dramatic fall in the incidence of the disease in non-immunized infants below the age of 12 months. In the five years 1948 to $195^{2}$ only nine deaths from diphtheria occurred in the first year of life. In 1953 no cases of the disease were notified in children unders one year.

The transfer of maternal antibody to the foetu位 has long been regarded as a serious obstacle to successful immunization before the age of sio months, and the practice in recent years of giving two successive, subcutaneous injections of 0.5 ce of diphtheria toxoid with an interval of four to siof weeks, during the second six months of life, is stip the routine procedure. Nevertheless, the recent demonstration, by several workers, that diphtheri刃 toxoid produces a satisfactory immunity in tho presence of a high antibody titre in the mother? 
blood has encouraged the belief that diphtheria immunization in the early months of life may, after all, be effective. Bousfield (1949-195I), after an extensive experience of immunizing young children against diphtheria, is fully convinced of the reliability of the procedure in young babies, despite the occasional presence of considerable amounts of anti-toxin transferred from the mother. Although such anti-toxin retarded the development of immunity it did not prevent its satisfactory development within a reasonable time. These conclusions correspond with those of Vahlquist (1949) and Greenberg and Fleming (I950, I95I), who demonstrated satisfactory antibody titres following immunization in the early months.

A highly purified diphtheria toxoid in buffered saline without added aluminium phosphate carrier has recently been proved effective (Holt, I949) and is now the prophylactic of choice, particularly during periods when there is any risk of poliomyelitis. It is given subcutaneously in three doses and this produces a higher conversion rate and a much higher degree of immunity than two doses of alum precipitated toxoid. It also has the advantage of being much less likely to cause a local reaction (Bousfield, I95I).

\section{Tetanus}

Tetanus is not strictly speaking an infectious disease, but its prevention should be considered when reviewing any policy of immunization. The average number of fatal cases in England and Wales in each year of the period $1943-5^{2}$ was 74.9 , but the true figure may be twice as great since, when both an injury and tetanus appear on a certificate, death is assigned to the injury. About one-third of the deaths occur between the ages of $I$ and $I_{5}$ years. Deaths from tetanus during the neonatal period have continued to fall during the last 30 years, but in older age groups the numbers have remained practically constant. The case incidence is uncertain as the disease is not notifiable, but the mortality rate is believed to be between 30 per cent. and $5 \circ$ per cent.

Prophylactic immunization with tetanus toxoid has proved effective and long-lasting. Moreover, the toxoid is more effective and safer than antitetanus serum in the protection of injured people who have been previously immunized. The incidence of tetanus in the United States Army in World War II was reduced to less than onethirtieth of the figure in World War I by routine active immunization and the disease almost eliminated.

The absence of a visible wound in a considerable proportion of cases of tetanus, and the much greater risk of serious reactions with anti-tetanus serum than with toxoid, are additional strong reasons for preferring active to passive immunization. Anti-tetanus serum occasionally causes anaphylaxis which is sometimes fatal, while serum sickness and rashes are common. The substitution of toxoid for anti-serum as a method of protection would eliminate these dangers. Anti-serum is more costly to produce than toxoid and much is used. It is estimated that one in every ten persons in this country receives an injection of it in their lifetime.

There is a strong case for routine active immunization against tetanus in childhood. The inclusion of tetanus toxoid as a component of a combined antigen preparation is now being practised on a large scale in Canada and the United States. The additional cost of combining this antigen with others is small. However, until a high proportion of the population have been actively immunized against tetanus anti-serum will continue to be given as a routine for immediate prophylaxis after injury. The technique of active tetanus immunization will be discussed later when considering combined immunization.

\section{Whooping Cough}

There has been a great decline in the mortality from whooping cough in the last 30 years and if now accounts for approximately I per cent. of the total infant mortality. Nevertheless, the decline if mortality from the other common infectious diseases has been even greater; for example, in 1952, an average year, I I I infants died from whooping cough in the first year of life. Although the incidence is highest in the toddler age group, 60 per cent. of the deaths occur in the first year; most of them in the first six months. The case mortality is greatest in the second and third months and declines thereafter. Whooping cough is also a common cause of permanent lung damage, particularly when contracted under the age of two years (Field, I949; Conway, 1951).

The pros and cons of immunization have been much debated. For a number of years authorities in the United States and Canada have been satisfied with the potency of certain makes of vaccine. In this country serious doubt was cast on the efficacy of vaccination by the unfavourable report of the Medical Research Council trials published in 1945 , but the poor results obtained were apparently due to unsatisfactory vaccines. The second Medical Research Council Report (I95I) of carefully controlled trials with vaccine, prepared according to the formula of Dr. Pearl Kendrick, proved the value of immunization when potent vaccines are used. It was found that the incidence of whooping cough was reduced to one-seventh and the disease was relatively benign. Comparable results are apparently being obtained in clinical trials at 
present being conducted at University College Hospital (Butler, 1954). Butler finds that good immunity is produced when immunization is started at the age of six weeks, and that there is a correlation between clinical immunity and agglutinin levels. Objectors to immunization cite the occasional occurrence of encephalitis, but as this is an even more frequent complication of the natural disease the slight risk of contracting encephalitis is not a valid reason for withholding the benefit of immunization, except possibly in allergic children. The efficacy of immunization has also been questioned because the disease may still be contracted, although in an attenuated form.

Haemophilus pertussis is a delicate organism which may readily lose its antigenic properties and so makes difficult the production of a reliable vaccine. British made Kendrick-type vaccines are at present being assayed in large-scale field trials by the Medical Research Council and the results of these trials will be available in a few months. If these trials give good results, and the manufacturers can guarantee the production of a reliable vaccine, whooping cough immunization should become a routine procedure in infancy, and it should be done as soon as satisfactory immunity can be induced, since the mortality varies inversely with age and almost half of the deaths occur under the age of six months. There is evidence that satisfactory immunity is usually obtained when the first of the three doses of vaccine is given at the age of 6 to 12 weeks. At present three 1 cc. doses of vaccine are given by subcutaneous injection at intervals of four to six weeks, but it is probable that vaccine will usually be administered in combination with other antigens in the future. Some believe that immunization in the early months may not be essential for the protection of the infant where a high proportion of older children have been immunized because the risk of exposure is much less.

\section{Combined Immunization}

For a number of years differing views have been held by immunologists regarding the effect of administering antigens in combination. Some years ago certain workers claimed to have demonstrated enhancement of response by combination, but Barr and Llewellyn-Jones in a recent publication (1953) regarded much of the evidence for synergy as unreliable. Whilst acknowledging that synergy does occur in certain circumstances, these workers confirmed that ' crowding out' of antibody formation in response to one antigen may occur by the 'saturation' of most of the foreign protein receptors by a previously administered antigen, or by a disproportionately high amount of another antigen given simultaneously.
Combined prophylactics containing diphtheria๊ and pertussis or diphtheria, tetanus and pertussis 3 antigens have been used on an increasing scale in? the United States and Canada for several years andare now being used extensively in this country. $\overrightarrow{A s}$ new combined prophylactic containing purifie of fluid diphtheria toxoid and $H$. pertussis vaccinछ has recently been developed by Holt and Bousfield (P.D. \& Co.). It has been used on a large scalep with good results. The antigenicity of the fluid diphtheria toxoid was consistently enhanced bys the suspension of pertussis vaccine. The Schiclo conversion rate after a single dose of plain diphtheria toxoid was 62.8 per cent., and after single dose of combined diphtheria-pertussis prophylactic 84.5 per cent. The results of a triat with this new combined prophylactic in young. babies have recently been published by Bousfield and Holt (1954). These workers gave three monthly subcutaneous injections of $\mathrm{I}$ cc. beginning at the age of two to five months irrespective of whether the infants were Schick positive or negae tive; when tested at 15 months only one of the 522 infants was Schick positive. This infant wasonly two months old when immunization begane Only four of 12 known home contacts developed whooping cough before the age of 15 months These workers also investigated the incidencesof whooping cough during the three and a half to fäin years before the start of school life in 586 childrep who received a combined diphtheria-pertussi. prophylactic, and in 242 who received diphtherig toxoid alone. In the 'combined' group 62.8 pee cent. of house contacts resisted whooping coug and 9.4 per cent. contracted it in moderate o. severe form, whereas in the control group (toxoid alone) only 5.2 per cent. resisted whooping coug $\vec{R}$. and 71.9 per cent. developed it in moderate op severe form. Thus the incidence of the disease. was 12 times greater and the moderate or sever form $7 \frac{1}{2}$ times greater in the control group. These are highly satisfactory figures which abundantlo justified the inclusion of the whooping cough vaccine used by Bousfield when immunizing against diphtheria in early infancy. Feldman (1954), who has just published his observations on the use of a triple diphtheria-tetanus-pertussis antigen, found a uniformly satisfactory response to all three antigens when the immunization course was coms menced at the age of three months.

The Medical Research Council are conducting field trials with combined diphtheria-pertussis antigen, and the Scottish Department of Healt under the guidance of the Medical Researcf Council is directing field trials of triple diphtheria pertussis-tetanus antigen. Combined antigens are being used extensively abroad, particularly in Canada and the United States, and although n 
large-scale controlled trials have been done in these countries there is a general impression that such antigens are effective. In the meantime, the use of combined antigens is likely to increase in this country if only because of their greater convenience.

When the method of combined immunization is employed a course of three subcutaneous injections of a triple mineral-free antigen containing diphtheria and tetanus toxoids and pertussis vaccine should be given in early infancy. The first dose should be given at the age of one to two months and the second and third at intervals of four to six weeks. The first fortifying dose of triple antigen should be given at the age of one year. The second fortifying dose consisting of diphtheria and tetanus toxoids should be given at the age of about five years. No further routine doses are necessary, but after intimate exposure to infection an additional dose of the appropriate antigen may be given as a further precaution. The risk of causing poliomyelitis by injecting alum-containing toxoids intramuscularly should be eliminated by giving mineralfree preparations subcutaneously.

Immunization procedures should be as simple as possible and the number of injections reduced to the minimum, for the number of defaulters varies directly with the number of injections in any course of immunization. Moreover, experience has shown that the success of immunization policies largely depends on Medical Officers of Health.

\section{Tuberculosis}

There is still a difference of opinion about the value of B.C.G. immunization in the prevention of tuberculosis, though most authorities believe it affords some protection. The bacillus CalmetteGuérin vaccine, which should not be given to anyone with a positive tuberculin skin test, induces a state of immunity after an interval of three to eight weeks. This immunity can be demonstrated by the development of a positive reaction to one of the tuberculin skin tests, of which the Mantoux is the most reliable. Immunity, as judged by tuberculin, lasts for lengths of time from a few years to ten or more. If it is desired to maintain immunity the tuberculin sensitivity must be tested from time to time, and when it becomes negative revaccination should be carried out. In Sweden B.C.G. vaccination is done routinely in the newborn and is repeated at school entry, school leaving and on enlistment in the armed forces if the tuberculin test has become negative.

Immunity from B.C.G. vaccination is weaker than that from spontaneous infection with virulent organisms. B.C.G. vaccination should not therefore be allowed to foster a false sense of security, nor should it lead to an abandonment of other preventive measures such as avoidance of the risk of heavy infection.

The present policy of the Ministry of Health is to advise B.C.G. immunization of contacts and children of school leaving age who are Mantoux negative. Immunization of the latter is being rapidly extended by local authorities to protect the vulnerable adolescent and young adult. The Ministry does not at present advocate routine immunization of the newborn.

\section{Poliomyelitis}

Research for a potent vaccine is being prosecuted with great vigour, and the results of the current large-scale trials in the United States are eagerly awaited.

\section{BIBLIOGRAPHY}

ANDERSON, S. G. (1954), Lancet, ii, 1 ro7.

BARR, M., LLEWELLYN-JONES, M. (1953), Brit. f. Exp. Path. 34, 12 .

BOUSFIELD, G. (1949), Lancet, i, I 100.

BOUSFIELD, G. (195I), Lancet, i, 1028 et seq.

BOUSFIELD, G., HOLT, L. B. (1954), Medical Officer, 92, 289.

BUTLER, N. R. (1954), Proc. Roy. Soc. Med., 47, 357.

CONWAY, D. J. (I95I), M.D. Thesis, Cambridge University Library.

ENDERS, J. F., WELLER, T. H., ROBBINS, F. C. (I949), Science, ro9, 85 .

ENDERS, J. F., PEEBLES, T. C. (1954), Proc. Soc. exp. Biol. N.Y., 86, 277.

FELDMAN, G. V. (1954), Arch. Dis. Childh., 29, 175.

FIELD, E. C. (1949), Pediatrics, 4, 231, 355.

GREENBERG, L., FLEMING, D. S. (1950), 7. Pediatrics, 36, 143

GREENBERG, L., FLEMING, D. S. (195I), Ibid, 39, 672.

HABEL, K. (1945), Pub. Health Rep., 60, 201.

HOLT, L. B. (1950), Developments in Diphtheria Prophylaxis, Heinemann, London.

JOHNSON, C. D., GOODPASTURE, E. W. (1934), F. Exper Med., 59, I

MEDICAL RESEARCH COUNCIL REPORT ON WHOOPING COUGH IMMUNISATION (1945), Brit. med. $\mathcal{f}$., ii, 206.

MEDICAL RESEARCH COUNCIL REPORT ON WHOOP-

ING COUGH IMMUNISATION (195I), Ibid, i, 1463.

WELleR, T. H. (1953), Proc. Soc. exp. Biol., N.Y., 83, 340.

VAHLQUIST, B. (1949), Lancet, i, 16.

\section{BACK NUMBERS OF THIS JOURNAL}

If any subscribers have copies, in good condition, of MARCH and APRIL I952, the Fellowship of Postgraduate Medicine, 60 Portland Place, London, W.r, will be glad to purchase them. 